\title{
Non-local, non-convex functionals converging to Sobolev norms
}

\author{
Haïm Brezis ${ }^{\mathrm{a}, \mathrm{b}, \mathrm{c}}$, Hoai-Minh Nguyen ${ }^{\mathrm{d}, *}$ \\ ${ }^{a}$ Department of Mathematics, Rutgers University, Hill Center, Busch Campus, 110 Frelinghuysen \\ Road, Piscataway, NJ 08854, USA \\ ${ }^{\mathrm{b}}$ Departments of Mathematics and Computer Science, Technion, Israel Institute of \\ Technology, 32.000 Haifa, Israel \\ ${ }^{\mathrm{c}}$ Laboratoire Jacques-Louis Lions, Sorbonne Universités, UPMC Université Paris-6, 4 place \\ Jussieu, 75005 Paris, France \\ d Department of Mathematics, EPFL SB CAMA, Station 8 CH-1015 Lausanne, Switzerland
}

\section{A R T I C L E I N F O}

\section{Article history:}

Received 3 September 2019

Accepted 5 September 2019

Communicated by Vicentiu Radulescu

\section{Keywords:}

Non-local

Non-convex

Pointwise convergence

$\Gamma$-convergence

Sobolev norms

\section{A B S T R A C T}

We study the pointwise convergence and the $\Gamma$-convergence of a family of nonlocal, non-convex functionals $\Lambda_{\delta}$ in $L^{p}(\Omega)$ for $p>1$. We show that the limits are multiples of $\int_{\Omega}|\nabla u|^{p}$. This is a continuation of our previous work where the case $p=1$ was considered.

(C) 2019 Elsevier Ltd. All rights reserved.

\section{Introduction and statement of the main results}

Assume that $\varphi:[0,+\infty) \rightarrow[0,+\infty)$ is defined at every point of $[0,+\infty), \varphi$ is continuous on $[0,+\infty)$ except at a finite number of points in $(0,+\infty)$ where it admits a limit from the left and from the right, and $\varphi(0)=0$. Let $\Omega \subset \mathbb{R}^{d}(d \geq 1)$ denote a domain which is either bounded and smooth, or $\Omega=\mathbb{R}^{d}$. Given a measurable function $u$ on $\Omega$, and a parameter $\delta>0$, we define the following non-local functionals, for $p>1$,

$$
\Lambda(u, \Omega):=\int_{\Omega} \int_{\Omega} \frac{\varphi(|u(x)-u(y)|)}{|x-y|^{p+d}} d x d y \quad \text { and } \quad \Lambda_{\delta}(u, \Omega):=\delta^{p} \Lambda(u / \delta, \Omega) .
$$

To simplify the notation, we will often delete $\Omega$ and write $\Lambda_{\delta}(u)$ instead of $\Lambda_{\delta}(u, \Omega)$.

As in [3], we consider the following four assumptions on $\varphi$ :

$$
\begin{gathered}
\varphi(t) \leq a t^{p+1} \text { in }[0,1] \text { for some positive constant } a, \\
\varphi(t) \leq b \text { in } \mathbb{R}_{+} \text {for some positive constant } b, \\
\varphi \text { is non-decreasing, }
\end{gathered}
$$

\footnotetext{
* Corresponding author at: Department of Mathematics, EPFL SB CAMA, Station 8 CH-1015 Lausanne, Switzerland. E-mail addresses: brezis@math.rutgers.edu (H. Brezis), hoai-minh.nguyen@epfl.ch (H.-M. Nguyen).
} 
and

$$
\gamma_{d, p} \int_{0}^{\infty} \varphi(t) t^{-(p+1)} d t=1, \text { where } \gamma_{d, p}:=\int_{\mathbb{S}^{d-1}}|\sigma \cdot e|^{p} d \sigma \text { for some } e \in \mathbb{S}^{d-1} .
$$

In this paper, we study the pointwise and the $\Gamma$-convergence of $\Lambda_{\delta}$ as $\delta \rightarrow 0$ for $p>1$. This is a continuation of our previous work [3] where the case $p=1$ was investigated in great details. Concerning the pointwise convergence of $\Lambda_{\delta}$, our main result is

Theorem 1. Let $d \geq 1$ and $p>1$. Assume (1.2), (1.3), and (1.5) (the monotonicity assumption (1.4) is not required here). We have

(i) There exists a positive constant $C_{p, \Omega}$ such that

$$
\Lambda_{\delta}(u, \Omega) \leq C_{p, \Omega} \int_{\Omega}|\nabla u|^{p} d x \quad \forall u \in W^{1, p}(\Omega), \forall \delta>0 ;
$$

moreover,

$$
\lim _{\delta \rightarrow 0} \Lambda_{\delta}(u, \Omega)=\int_{\Omega}|\nabla u|^{p} d x \quad \forall u \in W^{1, p}(\Omega) .
$$

(ii) Assume in addition that $\varphi$ satisfies (1.4). Let $u \in L^{p}(\Omega)$ be such that

$$
\liminf _{\delta \rightarrow 0} \Lambda_{\delta}(u, \Omega)<+\infty
$$

then $u \in W^{1, p}(\Omega)$.

Remark 1. Theorem 1 provides a characterization of the Sobolev space $W^{1, p}(\Omega)$ for $p>1$ :

$$
W^{1, p}(\Omega)=\left\{u \in L^{p}(\Omega) ; \liminf _{\delta \rightarrow 0} \Lambda_{\delta}(u)<+\infty\right\} .
$$

This fact is originally due to Bourgain and Nguyen $[1,4]$ when $\varphi=\hat{\varphi}_{1}:=c \mathbb{1}_{(1,+\infty)}$ for an appropriate constant $c$.

There are some similarities but also striking differences between the cases $p>1$ and $p=1$.

(a) First note a similarity. Let $p=1$ and $\varphi$ satisfy (1.2)-(1.4), and assume that $u \in L^{1}(\Omega)$ verifies

$$
\liminf _{\delta \rightarrow 0} \Lambda_{\delta}(u, \Omega)<+\infty
$$

then $u \in B V(\Omega)$ (see $[1,3])$.

(b) Next is a major difference. Let $p=1$. There exists $u \in W^{1,1}(\Omega)$ such that, for all $\varphi$ satisfying (1.2)-(1.4), one has

$$
\lim _{\delta \rightarrow 0} \Lambda_{\delta}(u, \Omega)=+\infty
$$

[3, Pathology 1]. In particular, (1.6) and (1.7) do not hold for $p=1$. An example in the same spirit was originally constructed by Ponce and is presented in [4]. Other pathologies occurring in the case $p=1$ can be found in [3, Section 2.2].

As we will see later, the proof of (1.6) involves the theory of maximal functions. The use of this theory was suggested independently by Nguyen [4] and Ponce and van Schaftingen (unpublished communication to the authors). The proof of (1.6) uses the same strategy as in [4].

We point out that assertion (ii) fails without the monotonicity condition (1.4) on $\varphi$. Here is an example e.g. with $\Omega=\mathbb{R}$. Let $\varphi=c \mathbb{1}_{(1,2)}$ for an appropriate, positive constant $c$. Let $u=\mathbb{1}_{(0,1)}$. One can easily check that $\Lambda_{\delta}(u)=0$ for $\delta \in(0,1 / 2)$ and it is clear that $u \notin W^{1, p}(\mathbb{R})$ for $p>1$. 
Concerning the $\Gamma$-convergence of $\Lambda_{\delta}$, our main result is

Theorem 2. Let $d \geq 1$ and $p>1$. Assume (1.2)-(1.5). Then

$$
\Lambda_{\delta}(\cdot, \Omega) \Gamma \text {-converges in } L^{p}(\Omega) \text { to } \Lambda_{0}(\cdot, \Omega):=\kappa \int_{\Omega}|\nabla \cdot|^{p} d x,
$$

as $\delta \rightarrow 0$, for some constant $\kappa$ which depends only on $p$ and $\varphi$, and verifies

$$
0<\kappa \leq 1 \text {. }
$$

Theorem 2 was known earlier when $\varphi=\hat{\varphi}_{1}[5,6]$.

The paper is organized as follows. Theorem 1 is proved in Section 2 and the proof of Theorem 2 is given in Section 3. Throughout the paper, we denote

$$
\varphi_{\delta}(t):=\delta^{p} \varphi(t / \delta) \text { for } p>1, \delta>0, t \geq 0 .
$$

\section{Proof of Theorem 1}

In view of the fact that $\liminf _{t \rightarrow+\infty} \varphi(t)>0$, assertion (1.8) is a direct consequence of [1, Theorem 1]; note that [1, Theorem 1] is stated for $\Omega=\mathbb{R}^{d}$ but the proof can be easily adapted to the case where $\Omega$ is bounded. It could also be deduced from Theorem 2 .

We now establish assertions (1.6) and (1.7). The proof consists of two steps.

Step 1: Proof of (1.6) and (1.7) when $\Omega=\mathbb{R}^{d}$ and $u \in W^{1, p}\left(\mathbb{R}^{d}\right)$. Replacing $y$ by $x+z$ and using polar coordinates in the $z$ variable, we find

$$
\int_{\mathbb{R}^{d}} d x \int_{\mathbb{R}^{d}} \frac{\varphi_{\delta}(|u(x)-u(y)|)}{|x-y|^{p+d}} d y=\int_{\mathbb{R}^{d}} d x \int_{0}^{+\infty} d h \int_{\mathbb{S}^{d-1}} \frac{\varphi_{\delta}(|u(x+h \sigma)-u(x)|)}{h^{p+1}} d \sigma .
$$

We have

$$
\begin{aligned}
& \int_{\mathbb{R}^{d}} d x \int_{0}^{+\infty} d h \int_{\mathbb{S}^{d-1}} \frac{\varphi_{\delta}(|u(x+h \sigma)-u(x)|)}{h^{p+1}} d \sigma \\
& =\int_{\mathbb{R}^{d}} d x \int_{0}^{+\infty} d h \int_{\mathbb{S}^{d-1}} \frac{\delta^{p} \varphi(|u(x+h \sigma)-u(x)| / \delta)}{h^{p+1}} d \sigma .
\end{aligned}
$$

Rescaling the variable $h$ gives

$$
\begin{aligned}
& \int_{\mathbb{R}^{d}} d x \int_{0}^{+\infty} d h \int_{\mathbb{S}^{d-1}} \frac{\delta^{p} \varphi(|u(x+h \sigma)-u(x)| / \delta)}{h^{p+1}} d \sigma \\
& \quad=\int_{\mathbb{R}^{d}} d x \int_{0}^{+\infty} d h \int_{\mathbb{S}^{d-1}} \frac{\varphi(|u(x+\delta h \sigma)-u(x)| / \delta)}{h^{p+1}} d \sigma .
\end{aligned}
$$

Combining (2.1), (2.2), and (2.3) yields

$$
\int_{\mathbb{R}^{d}} d x \int_{\mathbb{R}^{d}} \frac{\varphi_{\delta}(|u(x)-u(y)|)}{|x-y|^{d+p}} d y=\int_{\mathbb{R}^{d}} d x \int_{0}^{+\infty} d h \int_{\mathbb{S}^{d-1}} \frac{\varphi(|u(x+\delta h \sigma)-u(x)| / \delta)}{h^{p+1}} d \sigma .
$$

Note that

$$
\lim _{\delta \rightarrow 0} \frac{|u(x+\delta h \sigma)-u(x)|}{\delta}=|\langle\nabla u(x), \sigma\rangle| h \text { for a.e. }(x, h, \sigma) \in \mathbb{R}^{d} \times[0,+\infty) \times \mathbb{S}^{d-1} .
$$

Here and in what follows, $\langle.,$.$\rangle denotes the usual scalar product in \mathbb{R}^{d}$. Since $\varphi$ is continuous at 0 and on $(0,+\infty)$ except at a finite number of points, it follows that

$$
\begin{aligned}
& \lim _{\delta \rightarrow 0} \frac{1}{h^{p+1}} \varphi(|u(x+\delta h \sigma)-u(x)| / \delta)=\frac{1}{h^{p+1}} \varphi(|\langle\nabla u(x), \sigma\rangle| h) \\
& \quad \text { for a.e. }(x, h, \sigma) \in \mathbb{R}^{d} \times(0,+\infty) \times \mathbb{S}^{d-1} .
\end{aligned}
$$


Rescaling once more the variable $h$ gives

$$
\int_{0}^{\infty} d h \int_{\mathbb{S}^{d-1}} \frac{1}{h^{p+1}} \varphi(|\langle\nabla u(x), \sigma\rangle| h) d \sigma=|\nabla u(x)|^{p} \int_{0}^{\infty} \varphi(t) t^{-(p+1)} d t \int_{\mathbb{S}^{d-1}}|\langle\sigma, e\rangle|^{p} d \sigma ;
$$

here we have also used the obvious fact that, for every $V \in \mathbb{R}^{d}$, and for any fixed $e \in \mathbb{S}^{d-1}$,

$$
\int_{\mathbb{S}^{d-1}}|\langle V, \sigma\rangle|^{p} d \sigma=|V|^{p} \int_{\mathbb{S}^{d-1}}|\langle e, \sigma\rangle|^{p} d \sigma .
$$

Thus, by the normalization condition (1.5), we obtain

$$
\int_{\mathbb{R}^{d}} d x \int_{0}^{\infty} d h \int_{\mathbb{S}^{d-1}} \frac{1}{h^{p+1}} \varphi(|\langle\nabla u(x), \sigma\rangle| h) d \sigma=\int_{\mathbb{R}^{d}}|\nabla u|^{p} d x .
$$

Set

$$
\widetilde{\varphi}(t)=\left\{\begin{array}{cl}
a t^{p+1} & \text { for } t \in[0,1) \\
b & \text { for } t \in[1,+\infty)
\end{array}\right.
$$

Then

$$
\widetilde{\varphi} \text { is non-decreasing and } \varphi \leq \widetilde{\varphi} \text {. }
$$

Note that, for a.e. $(x, h, \sigma) \in \mathbb{R}^{d} \times(0,+\infty) \times \mathbb{S}^{d-1}$,

$$
\frac{|u(x+\delta h \sigma)-u(x)|}{\delta} \leq \frac{1}{\delta} \int_{0}^{h \delta}|\langle\nabla u(x+s \sigma), \sigma\rangle| d s \leq h M(\nabla u, \sigma)(x),
$$

where

$$
M(\nabla u, \sigma)(x):=\sup _{t>0} \frac{1}{t} \int_{0}^{t}|\langle\nabla u(x+s \sigma), \sigma\rangle| d s .
$$

Combining (2.4) and (2.10), we derive from (2.9) that

$$
\begin{aligned}
& \Lambda_{\delta}(u) \leq \int_{\mathbb{S}^{d-1}} \int_{\mathbb{R}^{d}} \int_{0}^{\infty} \frac{\widetilde{\varphi}(h|M(\nabla u, \sigma)(x)|)}{h^{p+1}} d h d x d \sigma \\
& =\int_{0}^{+\infty} \widetilde{\varphi}(t) t^{-(p+1)} d t \int_{\mathbb{S}^{d-1}} \int_{\mathbb{R}^{d}}|M(\nabla u, \sigma)(x)|^{p} d x d \sigma .
\end{aligned}
$$

We claim that, for $\sigma \in \mathbb{S}^{d-1}$,

$$
\int_{\mathbb{R}^{d}}|M(\nabla u, \sigma)(x)|^{p} d x \leq C_{p} \int_{\mathbb{R}^{d}}|\nabla u(x)|^{p} d x .
$$

For notational ease, we will only consider the case $\sigma=e_{1}$. By the theory of maximal functions (see e.g. [7]), one has, for $g \in L^{p}(\mathbb{R})$,

$$
\int_{\mathbb{R}}\left|\sup _{t>0} f_{\xi-t}^{\xi+t}\right| g(s)|d s|^{p} d \xi \leq C_{p} \int_{\mathbb{R}}|g(\xi)|^{p} d \xi .
$$

Using this inequality with $g\left(x_{1}\right)=\partial_{x_{1}} u\left(x_{1}, x^{\prime}\right)$ for $x^{\prime} \in \mathbb{R}^{d-1}$, we obtain

$$
\int_{\mathbb{R}}\left|M\left(\nabla u, e_{1}\right)\left(x_{1}, x^{\prime}\right)\right|^{p} d x_{1} \leq C_{p} \int_{\mathbb{R}}\left|\partial_{x_{1}} u\left(x_{1}, x^{\prime}\right)\right|^{p} d x_{1} .
$$

Integrating with respect to $x^{\prime}$ yields

$$
\int_{\mathbb{R}^{d}}\left|M\left(\nabla u, e_{1}\right)(x)\right|^{p} d x \leq C_{p} \int_{\mathbb{R}^{d-1}} \int_{\mathbb{R}}\left|\partial_{x_{1}} u\left(x_{1}, x^{\prime}\right)\right|^{p} d x_{1} d x^{\prime} \leq C_{p} \int_{\mathbb{R}^{d}}|\nabla u(x)|^{p} d x,
$$

and (2.12) follows. 
Using (2.12), we deduce from (2.11) that

$$
\Lambda_{\delta}(u) \leq C_{p, d} \int_{\mathbb{R}^{d}}|\nabla u|^{p} d x
$$

which is (1.6). From (2.6), (2.7), (2.8), and (2.10) we derive, using the dominated convergence theorem, that

$$
\lim _{\delta \rightarrow 0} \Lambda_{\delta}(u)=\int_{\mathbb{R}^{d}}|\nabla u|^{p} d x
$$

This completes Step 1 .

Step 2: Proof of (1.6) and (1.7) when $\Omega$ is bounded and $u \in W^{1, p}(\Omega)$. We first claim that

$$
\lim _{\delta \rightarrow 0} \Lambda_{\delta}(u)=\int_{\Omega}|\nabla u|^{p} \text { for } u \in W^{1, p}(\Omega) .
$$

Indeed, consider an extension of $u$ in $\mathbb{R}^{d}$ which belongs to $W^{1, p}\left(\mathbb{R}^{d}\right)$, and is still denoted by $u$. By the same method as in the case $\Omega=\mathbb{R}^{d}$, we have

$$
\lim _{\delta \rightarrow 0} \int_{\Omega} d x \int_{\mathbb{R}^{d}} \frac{\varphi_{\delta}(|u(x)-u(y)|)}{|x-y|^{p+d}} d y=\int_{\Omega}|\nabla u|^{p} d x
$$

and, for $D \Subset \Omega$ and $\varepsilon>0$,

$$
\lim _{\delta \rightarrow 0} \int_{D} d x \int_{B(x, \varepsilon)} \frac{\varphi_{\delta}(|u(x)-u(y)|)}{|x-y|^{p+d}} d y=\int_{D}|\nabla u|^{p} d x .
$$

Combining (2.14) and (2.15) yields (2.13).

We next show that

$$
\Lambda_{\delta}(u) \leq C_{p, \Omega} \int_{\Omega}|\nabla u|^{p} d x \text { for } u \in W^{1, p}(\Omega) .
$$

Without loss of generality, we may assume that $\int_{\Omega} u=0$. Consider an extension $U$ of $u$ in $\mathbb{R}^{d}$ such that

$$
\int_{\mathbb{R}^{d}}|\nabla U|^{p} d x \leq C_{p, \Omega} \int_{\Omega}|\nabla u|^{p} d x .
$$

Such an extension exists since $\Omega$ is smooth and $\int_{\Omega} u=0$, see, e.g., [2, Chapter 9]. Using the fact

$$
\Lambda_{\delta}(u, \Omega) \leq \Lambda_{\delta}\left(U, \mathbb{R}^{d}\right) \leq C_{p, d} \int_{\mathbb{R}^{d}}|\nabla U|^{p} d x
$$

we get (2.16). The proof is complete.

\section{Proof of Theorem 2}

We first recall the meaning of $\Gamma$-convergence. One says that $\Lambda_{\delta}(\cdot, \Omega) \stackrel{\Gamma}{\rightarrow} \Lambda_{0}(\cdot, \Omega)$ in $L^{p}(\Omega)$ as $\delta \rightarrow 0$ if

(G1) For each $g \in L^{p}(\Omega)$ and for every family $\left(g_{\delta}\right) \subset L^{p}(\Omega)$ such that $\left(g_{\delta}\right)$ converges to $g$ in $L^{p}(\Omega)$ as $\delta \rightarrow 0$, one has

$$
\liminf _{\delta \rightarrow 0} \Lambda_{\delta}\left(g_{\delta}, \Omega\right) \geq \Lambda_{0}(g, \Omega) .
$$

(G2) For each $g \in L^{p}(\Omega)$, there exists a family $\left(g_{\delta}\right) \subset L^{p}(\Omega)$ such that $\left(g_{\delta}\right)$ converges to $g$ in $L^{p}(\Omega)$ as $\delta \rightarrow 0$, and

$$
\limsup _{\delta \rightarrow 0} \Lambda_{\delta}\left(g_{\delta}, \Omega\right) \leq \Lambda_{0}(g, \Omega)
$$


Denote $Q$ the unit open cube, i.e., $Q=(0,1)^{d}$ and set

$$
U(x)=d^{-1 / 2} \sum_{j=1}^{d} x_{j} \text { in } Q,
$$

so that $|\nabla U|=1$ in $Q$.

In the following two subsections, we establish properties (G1) and (G2) where $\kappa$ is the constant defined by

$$
\kappa=\inf \liminf _{\delta \rightarrow 0} \Lambda_{\delta}\left(v_{\delta}, Q\right) .
$$

Here the infimum is taken over all families of functions $\left(v_{\delta}\right) \subset L^{p}(Q)$ such that $v_{\delta} \rightarrow U$ in $L^{p}(Q)$ as $\delta \rightarrow 0$.

\subsection{Proof of Property (G1)}

We begin with

Lemma 1. Let $d \geq 1, p>1, S$ be an open bounded subset of $\mathbb{R}^{d}$ with Lipschitz boundary, and let $g$ be an affine function. Then

$$
\inf \liminf _{\delta \rightarrow 0} \Lambda_{\delta}\left(g_{\delta}, S\right)=\kappa|\nabla g|^{p}|S|,
$$

where the infimum is taken over all families $\left(g_{\delta}\right) \subset L^{p}(S)$ such that $g_{\delta} \rightarrow g$ in $L^{p}(S)$ as $\delta \rightarrow 0$.

Proof. The proof of Lemma 1 is based on the definition of $\kappa$ in (3.1) and a covering argument. It is identical to the one of the first part of [3, Lemma 6$]$. The details are omitted.

The proof of Property (G1) for $p>1$ relies on the following lemma with roots in [6].

Lemma 2. Let $d \geq 1, p>1$, and $\varepsilon>0$. There exist two positive constants $\hat{\delta}_{1}, \hat{\delta}_{2}$ such that for every open cube $\widetilde{Q}$ which is an image of $Q$ by a dilation, for every $a \in \mathbb{R}^{d}$, every $b \in \mathbb{R}$, and every $h \in L^{p}(\widetilde{Q})$ satisfying

$$
f_{\widetilde{Q}}|h(x)-(\langle a, x\rangle+b)|^{p} d x \leq \hat{\delta}_{1}|a|^{p}|\widetilde{Q}|^{p / d},
$$

one has

$$
\Lambda_{\delta}(h, \widetilde{Q}) \geq(\kappa-\varepsilon)|a|^{p}|\widetilde{Q}| \text { for } \delta \in\left(0, \hat{\delta}_{2}|a||\widetilde{Q}|^{1 / d}\right) .
$$

Hereafter, as usual, we denote $f_{A} f=\frac{1}{|A|} \int_{A} f$.

Proof. By a change of variables, without loss of generality, it suffices to prove Lemma 2 in the case $\widetilde{Q}=Q$, $|a|=1$, and $b=0$. We prove this by contradiction. Suppose that this is not true. There exist $\varepsilon_{0}>0$, a sequence of measurable functions $\left(h_{n}\right) \subset L^{p}(Q)$, a sequence $\left(a_{n}\right) \subset \mathbb{R}^{d}$, and a sequence $\left(\delta_{n}\right)$ converging to 0 such that $\left|a_{n}\right|=1$,

$$
\int_{Q}\left|h_{n}(x)-\left\langle a_{n}, x\right\rangle\right|^{p} \leq \frac{1}{n}, \quad \text { and } \quad \Lambda_{\delta_{n}}\left(h_{n}, Q\right)<\kappa-\varepsilon_{0} .
$$

Without loss of generality, we may assume that $\left(a_{n}\right)$ converges to $a$ for some $a \in \mathbb{R}^{d}$ with $|a|=1$. It follows that $\left(h_{n}\right)$ converges to $\langle a,$.$\rangle in L^{p}(Q)$. Applying Lemma 1 with $S=Q$ and $g=\langle a, \cdot\rangle$, we obtain a contradiction. The conclusion follows.

The second key ingredient in the proof of Property (G1) is the following useful property of functions in $W^{1, p}\left(\mathbb{R}^{d}\right)$. 
Lemma 3. Let $d \geq 1, p>1$, and $u \in W^{1, p}\left(\mathbb{R}^{d}\right)$. Given $\varepsilon_{1}>0$, there exist a subset $B=B\left(\varepsilon_{1}\right)$ of Lebesgue points of $u$ and $\nabla u$, and an integer $\ell=\ell\left(\varepsilon_{1}\right) \geq 1$ such that

$$
\int_{\mathbb{R}^{d} \backslash B}|\nabla u|^{p} d x \leq \varepsilon_{1} \int_{\mathbb{R}^{d}}|\nabla u|^{p} d x
$$

and, for every open cube $Q^{\prime}$ with $\left|Q^{\prime}\right|^{1 / d} \leq 1 / \ell$ and $Q^{\prime} \cap B \neq \emptyset$, and for every $x \in Q^{\prime} \cap B$,

$$
\frac{1}{\left|Q^{\prime}\right|^{p}} f_{Q^{\prime}}|u(y)-u(x)-\langle\nabla u(x), y-x\rangle|^{p} d y \leq \varepsilon_{1}
$$

and

$$
|\nabla u(x)|^{p} \geq\left(1-\varepsilon_{1}\right) f_{Q^{\prime}}|\nabla u(y)|^{p} d y .
$$

Proof. We first recall the following property of $W^{1, p}\left(\mathbb{R}^{d}\right)$ functions (see e.g., [8, Theorem 3.4.2]): for a.e. $x \in \mathbb{R}^{d}$,

$$
\lim _{r \rightarrow 0} \frac{1}{r^{p}} f_{Q(x, r)}|u(y)-u(x)-\langle\nabla u(x), y-x\rangle|^{p} d y=0,
$$

where $Q(x, r):=x+(-r, r)^{d}$ for $x \in \mathbb{R}^{d}$ and $r>0$.

Given $n \in \mathbb{N}$, define, for a.e. $x \in \mathbb{R}^{d}$,

$$
\rho_{n}(x)=\sup \left\{\frac{1}{r^{p}} f_{Q(x, r)}|u(y)-u(x)-\langle\nabla u(x), y-x\rangle|^{p} d y ; r \in(0,1 / n)\right\}
$$

and

$$
\tau_{n}(x)=\sup \left\{f_{Q(x, r)}|\nabla u(y)-\nabla u(x)|^{p} d y ; r \in(0,1 / n)\right\} .
$$

Note that, by (3.8), $\rho_{n}(x) \rightarrow 0$ for a.e. $x \in \mathbb{R}^{d}$ as $n \rightarrow+\infty$. We also have, $\tau_{n}(x) \rightarrow 0$ for a.e. $x \in \mathbb{R}^{d}$ as $n \rightarrow+\infty$ (and in fact at every Lebesgue point of $\nabla u$ ). For $m \geq 1$, set

$$
D_{m}=\left\{x \in(-m, m)^{d} ; x \text { is a Lebesgue point of } u \text { and } \nabla u \text {, and }|\nabla u(x)| \geq 1 / m\right\} .
$$

Since

$$
\lim _{m \rightarrow+\infty} \int_{\mathbb{R}^{d} \backslash D_{m}}|\nabla u|^{p} d x=0,
$$

there exists $m \geq 1$ such that

$$
\int_{\mathbb{R}^{d} \backslash D_{m}}|\nabla u|^{p} d x \leq \frac{\varepsilon_{1}}{2} \int_{\mathbb{R}^{d}}|\nabla u|^{p} d x .
$$

Fix such an $m$. By Egorov's theorem, there exists a subset $B \subset D_{m}$ such that $\left(\rho_{n}\right)$ and $\left(\tau_{n}\right)$ converge to 0 uniformly on $B$, and

$$
\int_{D_{m} \backslash B}|\nabla u|^{p} d x \leq \frac{\varepsilon_{1}}{2} \int_{\mathbb{R}^{d}}|\nabla u|^{p} d x .
$$

Combining (3.11) and (3.12) yields (3.5).

By the triangle inequality, we have, for every non-empty, open cube $Q^{\prime}$ and a.e. $x \in \mathbb{R}^{d}$ (in particular for $\left.x \in Q^{\prime} \cap B\right)$,

$$
\left(f_{Q^{\prime}}|\nabla u(y)|^{p} d y\right)^{1 / p} \leq\left(f_{Q^{\prime}}|\nabla u(y)-\nabla u(x)|^{p} d y\right)^{1 / p}+|\nabla u(x)| \leq \frac{|\nabla u(x)|}{\left(1-\varepsilon_{1}\right)^{1 / p}}
$$

provided

$$
\left(f_{Q^{\prime}}|\nabla u(y)-\nabla u(x)|^{p} d y\right)^{1 / p} \leq\left(\frac{1}{\left(1-\varepsilon_{1}\right)^{1 / p}}-1\right) 1 / m \quad \text { and } \quad|\nabla u(x)| \geq 1 / m .
$$


Since $\left(\rho_{n}\right)$ and $\left(\tau_{n}\right)$ converge to 0 uniformly on $B$ and $|\nabla u(x)| \geq 1 / m$ for $x \in B$, it follows from (3.13) that there exists an $\ell \geq 1$ such that (3.6) and (3.7) hold when $\left|Q^{\prime}\right|^{1 / d} \leq 1 / \ell$ and $Q^{\prime} \cap B \neq \emptyset$, and $x \in Q^{\prime} \cap B$. The proof is complete.

We are ready to give the

Proof of Property (G1). We only consider the case $\Omega=\mathbb{R}^{d}$. The other case can be handled as in [3] and is left to the reader. We follow the same strategy as in [6].

In order to establish Property (G1), it suffices to prove that

$$
\liminf _{k \rightarrow+\infty} \Lambda_{\delta_{k}}\left(g_{k}, \mathbb{R}^{d}\right) \geq \kappa \int_{\mathbb{R}^{d}}|\nabla g|^{p} d x
$$

for every $g \in L^{p}\left(\mathbb{R}^{d}\right),\left(\delta_{k}\right) \subset \mathbb{R}_{+}$and $\left(g_{k}\right) \subset L^{p}\left(\mathbb{R}^{d}\right)$ such that $\delta_{k} \rightarrow 0$ and $g_{k} \rightarrow g$ in $L^{p}\left(\mathbb{R}^{d}\right)$.

Without loss of generality, we may assume that $\liminf _{k \rightarrow+\infty} \Lambda_{\delta_{k}}\left(g_{k}, \mathbb{R}^{d}\right)<+\infty$. It follows from [6] that $g \in W^{1, p}\left(\mathbb{R}^{d}\right)$. Fix $\varepsilon>0$ (arbitrary) and let $\hat{\delta}_{1}$ be the positive constant in Lemma 2. Set, for $m \geq 1$,

$$
A_{m}=\left\{x \in \mathbb{R}^{d} ; x \text { is a Lebesgue point of } g \text { and } \nabla g, \text { and }|\nabla g(x)| \leq 1 / m\right\} .
$$

Since

$$
\lim _{m \rightarrow+\infty} \int_{A_{m}}|\nabla g|^{p} d x=0
$$

there exists $m \geq 1$ such that

$$
\int_{A_{m}}|\nabla g|^{p} d x \leq \frac{\varepsilon}{2} \int_{\mathbb{R}^{d}}|\nabla g|^{p} d x
$$

Fix such an integer $m$. By Lemma 3 applied to $u=g$ and $\varepsilon_{1}=\min \left\{\varepsilon / 2, \delta_{1} /(2 m)^{p}\right\}$, there exist a subset $B$ of Lebesgue points of $g$ and $\nabla g$, and a positive integer $\ell$ such that

$$
\int_{\mathbb{R}^{d} \backslash B}|\nabla g|^{p} d x \leq \varepsilon_{1} \int_{\mathbb{R}^{d}}|\nabla g|^{p} d x \leq \frac{\varepsilon}{2} \int_{\mathbb{R}^{d}}|\nabla g|^{p} d x,
$$

and for every open cube $Q^{\prime}$ with $\left|Q^{\prime}\right|^{1 / d} \leq 1 / \ell$ and $Q^{\prime} \cap B \neq \emptyset$, and, for every $x \in Q^{\prime} \cap B$,

$$
\frac{1}{\left|Q^{\prime}\right|^{p / d}} f_{Q^{\prime}}|g(y)-g(x)-\langle\nabla g(x), y-x\rangle|^{p} d y \leq \varepsilon_{1} \leq \hat{\delta}_{1} /(2 m)^{p}
$$

and

$$
|\nabla g(x)|^{p}\left|Q^{\prime}\right| \geq\left(1-\varepsilon_{1}\right) \int_{Q^{\prime}}|\nabla g|^{p} d y \geq(1-\varepsilon) \int_{Q^{\prime}}|\nabla g|^{p} d y .
$$

Fix such a set $B$ and such an integer $\ell$. Set

$$
B_{m}:=B \backslash A_{m}
$$

Since $\mathbb{R}^{d} \backslash\left(B \backslash A_{m}\right) \subset\left(\mathbb{R}^{d} \backslash B\right) \cup A_{m}$, it follows that

$$
\int_{\mathbb{R}^{d} \backslash B_{m}}|\nabla g|^{p} d x=\int_{\mathbb{R}^{d} \backslash\left(B \backslash A_{m}\right)}|\nabla g|^{p} d x \leq \int_{\mathbb{R}^{d} \backslash B}|\nabla g|^{p} d x+\int_{A_{m}}|\nabla g|^{p} d x .
$$

We deduce from (3.15) and (3.16) that

$$
\int_{\mathbb{R}^{d} \backslash B_{m}}|\nabla g|^{p} d x \leq \varepsilon \int_{\mathbb{R}^{d}}|\nabla g|^{p} d x .
$$


Set $P_{\ell}=\frac{1}{\ell} \mathbb{Z}^{d}$. Let $\boldsymbol{\Omega}_{\ell}$ be the collection of all open cubes with side length $1 / \ell$ whose vertices belong to $P_{\ell}$ and denote

$$
\mathbf{J}_{\ell}=\left\{Q^{\prime} \in \boldsymbol{\Omega}_{\ell} ; Q^{\prime} \cap B_{m} \neq \emptyset\right\} .
$$

Take $Q^{\prime} \in \mathbf{J}_{\ell}$ and $x \in Q^{\prime} \cap B_{m}$. Since $g_{k} \rightarrow g$ in $L^{p}\left(Q^{\prime}\right)$, from (3.17), we obtain, for large $k$,

$$
\frac{1}{\left|Q^{\prime}\right|^{p / d}} f_{Q^{\prime}}\left|g_{k}(y)-g(x)-\langle\nabla g(x), y-x\rangle\right|^{p} d y<\hat{\delta}_{1} / m^{p} \leq \hat{\delta}_{1}|\nabla g(x)|^{p},
$$

since $|\nabla g(x)| \geq 1 / m$ for $x \in B_{m} \subset \mathbb{R}^{d} \backslash A_{m}$. Next, we apply Lemma 2 with $\widetilde{Q}=Q^{\prime}, h=g_{k}, a=\nabla g(x)$, $b=g(x)$, and large $k$; we have

$$
\Lambda_{\delta}\left(g_{k}, Q^{\prime}\right) \geq(\kappa-\varepsilon)|\nabla g(x)|^{p}\left|Q^{\prime}\right| \text { for } \delta \in\left(0, \hat{\delta}_{2}|\nabla g(x)|^{p}\left|Q^{\prime}\right|^{1 / d}\right),
$$

which implies, by (3.18),

$$
\liminf _{k \rightarrow+\infty} \Lambda_{\delta_{k}}\left(g_{k}, Q^{\prime}\right) \geq(\kappa-\varepsilon)(1-\varepsilon) \int_{Q^{\prime}}|\nabla g|^{p} d y
$$

Since

$$
\liminf _{k \rightarrow+\infty} \Lambda_{\delta_{k}}\left(g_{k}, \mathbb{R}^{d}\right) \geq \sum_{Q^{\prime} \in \mathbf{J}_{\ell}} \liminf _{k \rightarrow+\infty} \Lambda_{\delta}\left(g_{k}, Q^{\prime}\right)
$$

it follows from (3.20) that

$$
\begin{aligned}
\liminf _{k \rightarrow+\infty} \Lambda_{\delta_{k}}\left(g_{k}, \mathbb{R}^{d}\right) & \geq(\kappa-\varepsilon)(1-\varepsilon) \sum_{Q^{\prime} \in \mathbf{J}_{\ell}} \int_{Q^{\prime}}|\nabla g|^{p} d x \\
& \geq(\kappa-\varepsilon)(1-\varepsilon) \int_{B_{m}}|\nabla g|^{p} d x \stackrel{(3.19)}{\geq}(\kappa-\varepsilon)(1-\varepsilon)^{2} \int_{\mathbb{R}^{d}}|\nabla g|^{p} d x
\end{aligned}
$$

in the second inequality, we have used the fact $B_{m}$ is contained in $\bigcup_{Q^{\prime} \in \mathbf{J}_{\ell}} Q^{\prime}$ up to a null set. Since $\varepsilon>0$ is arbitrary, one has

The proof is complete.

$$
\liminf _{k \rightarrow+\infty} \Lambda_{\delta_{k}}\left(g_{k}, \mathbb{R}^{d}\right) \geq \kappa \int_{\mathbb{R}^{d}}|\nabla g|^{p} d x
$$

\subsection{Proof of Property (G2)}

The proof of Property (G2) for $p>1$ is the same as the one for $p=1$ given in [3]. The details are omitted.

\section{Acknowledgments}

This work was completed during a visit of H.-M. Nguyen at Rutgers University. He thanks H. Brezis for the invitation and the Department of Mathematics for its hospitality.

\section{References}

[1] J. Bourgain, H.-M. Nguyen, A new characterization of Sobolev spaces, C. R. Acad. Sci. Paris 343 (2006) 75-80.

[2] H. Brezis, Functional Analysis, Sobolev Spaces and Partial Differential Equations, Springer, 2010.

[3] H. Brezis, H.-M. Nguyen, Non-local functionals related to the total variation and applications in image processing, Ann. PDE 4 (2018) 77.

[4] H.-M. Nguyen, Some new characterizations of Sobolev spaces, J. Funct. Anal. 237 (2006) 689-720.

[5] H.-M. Nguyen, $\Gamma$-Convergence and Sobolev norms, C. R. Acad. Sci. Paris 345 (2007) 679-684.

[6] H.-M. Nguyen, $\Gamma$-Convergence, Sobolev norms, and BV functions, Duke Math. J. 157 (2011) $495-533$.

[7] E. Stein, Harmonic Analysis: Real-Variable Methods, Orthogonality, and Oscillatory Integrals, in: Princeton Mathematical Series, vol. 43, Princeton University Press, Princeton, 1993.

[8] W. Ziemer, Weakly Differentiable Functions. Sobolev Spaces and Functions of Bounded Variation, in: Graduate Texts in Mathematics, vol. 120, Springer-Verlag, New York, 1989. 